\title{
Delayed Bleeding after Percutaneous Liver Biopsy
}

\author{
Yaniv Dotan ${ }^{1}$, Matthias Carlebach ${ }^{2}$, Eli Zuckerman ${ }^{3}$, Musa Maruf $^{4}$, Elad Schiff ${ }^{1}$ \\ ${ }^{1}$ Department of Internal Medicine, Bnai Zion Medical Center, Haifa, Israel \\ 2Department of Gastroenterology, Bnai Zion Medical Center, Haifa, Israel \\ ${ }^{3}$ Liver Unit, Lady Davis Carmel Medical Center, Haifa, Israel \\ ${ }^{4}$ Department of Emergency Medicine, Bnai Zion Medical Center, Haifa, Israel
}

Received: $14 / 12 / 2015$

Accepted: 29/12/2015

Published: $18 / 01 / 2016$

How to cite this article: Dotan Y, Carlebach M, Zuckerman E, Maruf M, Schiff E. Delayed bleeding after percutaneous liver biopsy. EJCRIM 2016;3:doi:10.12890/2016_000359

Conflicts of Interests: The authors declare that there are no competing interests.

This article is licensed under a Commons Attribution Non-Commercial 4.0 License

\section{ABSTRACT}

Percutaneous liver biopsy (PLB) is a common procedure in patients with liver disease. Bleeding after PLB is rare, with an incidence of $0.35 \%$. Most bleeding complications present within $24 \mathrm{~h}$ after biopsy. A 56-year-old woman was admitted to our hospital due to severe and sudden right upper quadrant (RUQ) abdominal pain 10 days after ultrasound (US)-guided PLB. CT study revealed both intrahepatic and intraperitoneal bleeding, and $\mathrm{Hb}$ levels decreased by $3.2 \mathrm{~g} / \mathrm{dl}$ within a few hours. Such a prolonged delay in PLB-related bleeding has not been previously described in the medical literature.

\section{LEARNING POINTS}

- Bleeding after liver biopsy is very rare, with an incidence of $0.35 \%$.

- Approximately $95 \%$ of bleeding complications occur within $24 \mathrm{~h}$.

- Physicians should be aware of rare delayed presentation in the days following liver biopsy.

\section{KEYWORDS}

Percutaneous liver biopsy; cirrhosis; bleeding.

\section{INTRODUCTION}

PLB is a common procedure in patients with liver disease. However, bleeding after PLB is rare, with incidence of 0.35\%. Approximately $95 \%$ of bleeding complications present within $24 \mathrm{~h}$ after biopsy ${ }^{[1]}$. We describe a rare case of delayed intraperitoneal and subcapsular bleeding 10 days after biopsy, which was treated conservatively. Complication rates, patient presentation, risk factors for bleeding, and surveillance time after biopsy are discussed briefly.

\section{CASE REPORT}

A 57-year-old woman was admitted to our hospital due to severe, sudden and sharp RUQ abdominal pain. The patient had a history of primary biliary cirrhosis (PBC), systemic lupus erythematosus and lichen planus. She had undergone US-guided PLB for staging of PBC 10 days before this admission. The biopsy procedure had been uneventful, and the laboratory values on the day of biopsy were within normal limits with INR 0.99, PTT $29.9 \mathrm{sec}$ and platelets 677×109/I. One passage PLB had been performed with a Hepafix (Braun) 15-gauge (1.8 $\mathrm{mm}$ ) needle. Following the biopsy, the patient had been supervised for $4 \mathrm{~h}$, was haemodynamically stable, did not report any pain, and was 
discharged home. The biopsy specimen revealed a non-cirrhotic liver. The patient denied having any abdominal pain or abdominal trauma in the days following biopsy. At presentation her vital signs were normal. Physical examination was normal other than RUQ abdominal tenderness. Laboratory studies revealed a haemoglobin $(\mathrm{Hb})$ level of $12 \mathrm{~g} / \mathrm{dl}$, no leukocytosis or thrombocytosis, INR 1.07, PTT 26 sec, normal electrolytes and renal function, normal bilirubin levels and elevated liver enzymes with ALP 113 U/I, GGT 269 U/I, ALT 202 U/I and AST $275 \mathrm{U} / \mathrm{I}$. Chest and abdominal x-rays were normal, and abdominal US showed a normal-sized liver with hyperechogenic areas in the right lobe suspected to be haematomas (Fig. 1).

The patient underwent an abdominal and pelvic computed tomography (CT) scan which demonstrated a large haematoma in the right lobe of the liver and a large amount of intraperitoneal fluid (Fig. 2).

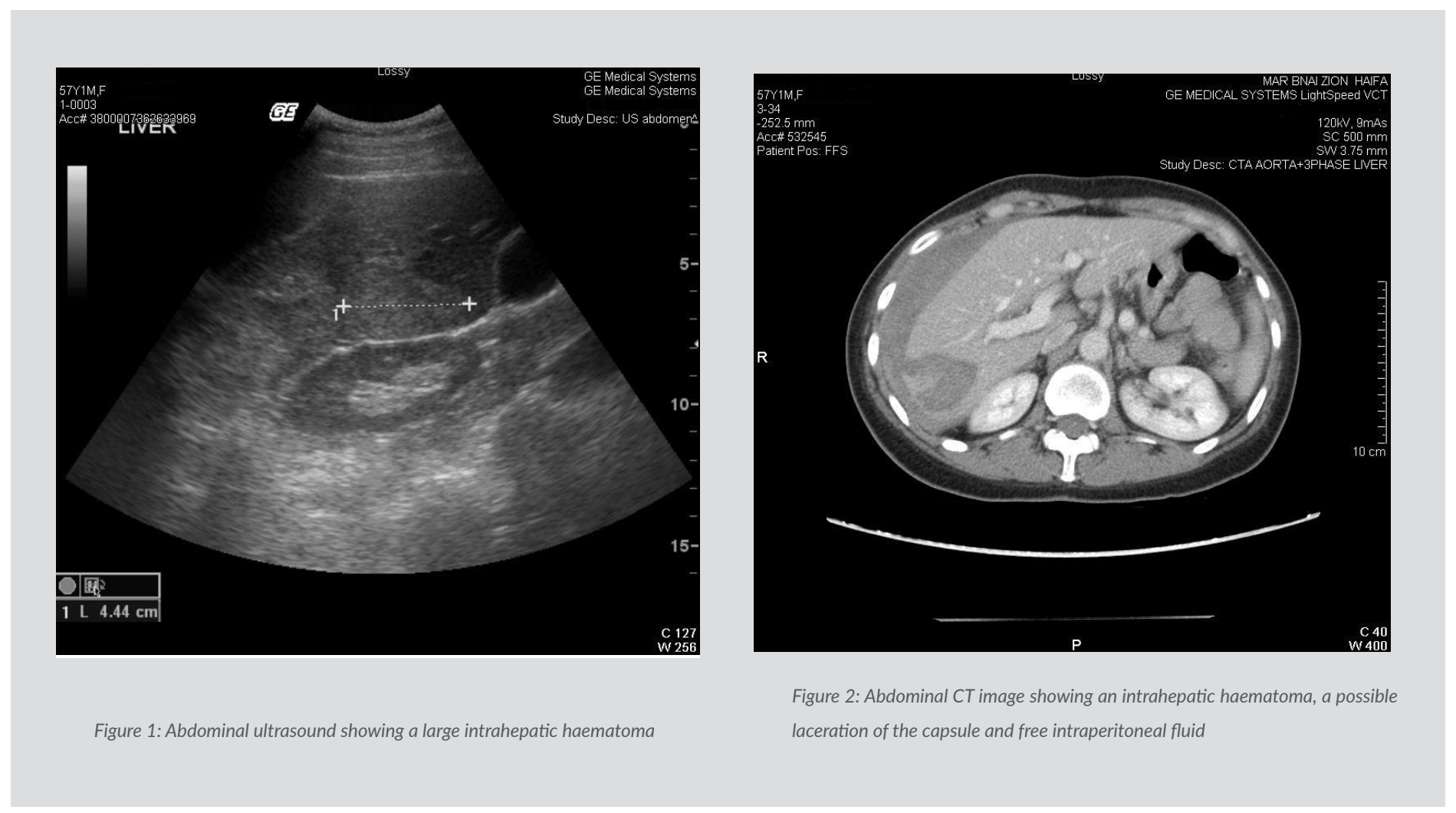

The patient was admitted and in light of haemodynamic stability was managed conservatively with frequent monitoring of vital signs and laboratory tests. Several hours after admission the $\mathrm{Hb}$ level decreased by $3.2 \mathrm{~g} / \mathrm{dl}$ to $8.8 \mathrm{~g} / \mathrm{dl}$. The patient was transfused with two units of packed red blood cells. Following blood transfusion, $\mathrm{Hb}$ levels remained stable at $10.5 \mathrm{~g} / \mathrm{dl}$.

From admission to discharge the patient was haemodynamically stable and reported relief of abdominal pain. $\mathrm{Hb}$ levels did not decrease, serial US examinations showed the haematoma in the right lobe of the liver did not increase in size, and Doppler imaging did not show any blood flow.

The patient was discharged home on day 7 of hospitalization.

\section{DISCUSSION}

PLB is a standard procedure for patients with chronic liver disease. US-guided PLB is considered generally safe and has a very low complication rate. In a large population-based study which included 4,275 patients between the years 1994 and 2002, 32 patients (0.75\%) had significant biopsy-related complications, and $15(0.35 \%)$ patients had bleeding. In this large study, six patients $(0.14 \%)$ died of complications due to bleeding, all of whom had malignancies ${ }^{[1]}$.

Most of the bleeding complications occur during the first $24 \mathrm{~h}$ after PLB: in a nationwide survey in France, $60 \%$ of bleeding complications 
occurred within $2 \mathrm{~h}, 82 \%$ in the first $10 \mathrm{~h}$, and $96 \%$ within $24 \mathrm{~h}$ after PLB ${ }^{2}$. According to the literature review we conducted, the report presented here is the most significantly delayed PLB-related bleeding case described so far.

Post-biopsy pain occurs in 30-50\% of patients after PLB and indicates transient localized discomfort at the biopsy site or a small amount of blood or bile, locally stretching the liver capsule. Usually the pain is mild to moderate in contrast to the severe sharp pain described by patients with major bleeding complications. However, it may be difficult to identify patients developing a major bleeding complication using clinical judgment alone.

PLB is carried out as an inpatient or outpatient procedure. In a large study of 3,214 patients comparing surveillance times after biopsy, no significant differences in major complications were observed between the groups at 1, 4 and $6 \mathrm{~h}$ after biopsy ${ }^{[3]}$.

The American Association for the Study of Liver Diseases (AASLD) published a position paper for liver biopsy in 2009. Regarding postbiopsy surveillance, the AASLD states that 'vital signs must be frequently monitored (at least every 15 minutes for the first hour) after liver biopsy (Class I, Level C). The recommended observation time after biopsy is between 2 to 4 hours and will vary depending on local expertise and practice (Class I, Level B) ${ }^{[4]}$.

Factors associated with an increased risk of bleeding include patient risk factors such as bleeding disorders, advanced age, cirrhosis, malignancy, renal failure and ascites. Technical risk factors include high numbers of needle passages, the use of a large needle, the use of an automatic needle, an inexperienced operator and the use of a blind technique. Our patient had none of the above personal or technical risk factors.

Our assumption is that the patient had minor intrahepatic bleeding which was asymptomatic and contained by the liver capsule, but on day 10 after biopsy, the liver capsule was torn abruptly and the blood previously contained in the capsule spilled into the peritoneal space, allowing additional bleeding from the damaged liver vessels to occur. An additional factor may have been premature clot dissolution due to liver disease-associated hyperfibrinolysis, which has been proposed as playing a role in some patients, especially those with delayed bleeding, although this has not been extensively studied ${ }^{[5]}$. This issue is even more complicated due to the fact that impaired haemostasis in patients with chronic liver disease is not properly reflected by conventional coagulation parameters.

In summary, we described a rare presentation of delayed bleeding after US-guided PLB. Physicians should be aware of such a presentation and instruct patients to immediately report any abdominal pain in the days following PLB.

\section{REFERENCES}

1. Myers PR, Fong A, Shaheen AA. Utilization rates, complications and costs of percutaneous liver biopsy: a population based study including 4275 biopsies. Liver Int 2008;28:705712.

2. Cardinal J-F, Rufat P, Degos F. Practices of liver biopsy in France: results of a nationwide survey. For the Group of Epidemiology of the French Association for the Study of the Liver. Hepatology 2000;32:477-481.

3. Firpi RJ, Soldevila PC, Abdelmalek MF, Morelli G, Judah J, Nelson DR. Short recovery time after percutaneous liver biopsy: should we change our current practice? Clin Gastroenterol Hepatol 2005;3:926-929.

4. Rockey DC, Caldwell SH, Goodman ZD, Nelson RC, Smith AD. Liver biopsy. AASLD position paper. Hepatology 2009;49:1017-1044.

5. Caldwell SH, Hoffman M, Lisman T, Macik BG, Northup PG, Reddy KR, et al. Coagulation disorders and hemostasis in liver disease: pathophysiology and critical assessment of current management. Hepatology 2006;44:1039-1046. 